\title{
Newly Diagnosed Crohn's Disease Treated with Standard Care or Enteral Nutrition: Psychological Outcomes over 6 Months
}

\author{
Catherine L. Wall ${ }^{a}$ Andrew M. McCombie ${ }^{b}$ Richard B. Gearry ${ }^{c}$ Andrew S. Day ${ }^{a}$ \\ a Department of Pediatrics, University of Otago, Christchurch, New Zealand; \\ ${ }^{b}$ Department of Medicine and Surgery, University of Otago, Christchurch, New Zealand; \\ 'Department of Medicine, University of Otago, Christchurch, New Zealand
}

\section{Keywords}

Enteral nutrition - Crohn's disease · Quality of life · Anxiety ·

Depression P Psychological outcome

\begin{abstract}
Background and Aim: Impaired health-related quality of life and higher rates of anxiety and depression are observed in patients with Crohn's disease. This single center study aimed to describe and compare longitudinal psychological outcomes of newly diagnosed Crohn's disease patients treated with standard care or enteral nutrition to induce remission. Methods: Two separate cohorts of patients were recruited from one center and treated with standard care $(n=22)$ or enteral nutrition $(n=18)$ to induce disease remission. Disease activity, quality of life, anxiety, and depression were assessed at diagnosis and month 6. Results: At baseline, quality of life $(n=40)$ was not correlated with disease activity; symptoms of anxiety and depression were present in 15 $(38 \%)$ and 5 (13\%) of the 40 patients, respectively. Significant improvements in quality of life $(p<0.0001)$ and anxiety scores $(p=0.002)$ were observed after 2 weeks of exclusive enteral nutrition, and at month 6 significant improvements in all psychological outcomes were observed. At month 6,
\end{abstract}

patients who received standard care had a significant improvement in quality of life $(p=0.004)$, but not in anxiety or depression scores, and there were no differences in psychological outcomes between the treatment groups. Overall, $34 / 40$ (85\%) patients were in clinical remission at month 6, and a clinically significant quality of life score was achieved by $31 / 40$ (78\%). Conclusions: Patient health-related quality of life and symptoms of anxiety and depression improved as disease activity improved, and 6 months after diagnosis psychological outcomes were similar in patients treated with standard care or enteral nutrition to initially induce remission.

(c) 2019 S. Karger AG, Basel

\section{Introduction}

It is well established that many patients with Crohn's disease $(C D)$ have poorer health-related quality of life (HRQOL) than the general population $[1,2]$. The determinants of HRQOL are varied and may include disease treatments, disease activity, and hospitalization [3]. Drug treatments affect HRQOL of patients with inflammatory bowel disease (IBD) variably. Biological treatments are

\section{KARGER}

(c) 2019 S. Karger AG, Basel

E-Mail karger@karger.com

www.karger.com/iid
Andrew S. Day

Department of Pediatrics, University of Otago

PO Box 4345

Christchurch 8140 (New Zealand)

E-Mail andrew.day@ otago.ac.nz 
used to induce disease remission, and as a maintenance treatment they are associated with sustained improvement in IBD-specific quality of life $[4,5]$, whereas improvements in symptoms on corticosteroids do not always correlate with improved quality of life [6-8].

Patients with $\mathrm{CD}$ also have higher rates of anxiety and depression than the general population $[9,10]$. Previous literature suggests that anxiety is present in up to $40 \%$ of adults with CD $[9,11-13]$ and that up to $10 \%$ suffer from depression [11, 13-15]. Active $C D$ is associated with greater symptoms of anxiety and depression $[13,14,16$, 17], and the achievement of disease remission mostly results in a reduction in symptoms of anxiety and depression [10]. There is some evidence that the presence of major depression, or high levels of anxiety, is associated with a poorer treatment outcome [18]. No studies have documented changes in anxiety and depression using the Hospital Anxiety and Depression Scale (HADS) subsequent to treatment with enteral nutrition (EN) in children or adults.

Few studies have assessed HRQOL in adults with CD using EN. The use of exclusive EN (EEN) to induce disease remission is associated with improved HRQOL in children, even when a nasogastric tube is required to administer the feed [19]. Similarly, Japanese adults with CD in remission who were using a half EN diet to maintain disease remission had IBD questionnaire (IBDQ) scores similar to those on a free diet [20]. The impact of EN treatment on HRQOL has not been assessed in western adults.

The aim of the current study was to document the baseline psychological characteristics of adults with newly diagnosed CD, evaluate the impact of EN therapies on HRQOL, anxiety, and depression, and compare changes with those observed in patients receiving induction of remission standard care. It was hypothesized that EN therapies are associated with improved psychological outcomes in adults with active $\mathrm{CD}$, but that at 6 months outcomes would be similar to those who had received standard care induction of remission treatments.

\section{Methods}

\section{Patient Recruitment and Treatments}

Two patient cohorts were recruited from the Christchurch Hospital or private clinics in Christchurch, New Zealand. A standard care group was recruited between April 2011 and April 2013, and an EN group was recruited between May 2013 and December 2015. Ethical approval for the enteral nutrition study was given by the New Zealand Northern B Health and Disability Ethics Com- mittee (ethics reference 13/NTB/11), and the pilot clinical trial was registered with the Australia New Zealand Clinical Trial Registry (trial No. 363665). Ethical approval for the standard care study was given by the New Zealand Upper South A Regional Ethics Committee.

\section{Standard Care Group}

The standard care group has been described elsewhere [21]; in brief, patients aged at least 18 years were recruited either in the endoscopy suite at the time of diagnosis or by telephone within 48 $\mathrm{h}$ of confirmation of the $\mathrm{CD}$ diagnosis. Patients in the standard care group took standard care treatments for active disease and maintenance of remission as prescribed by their gastroenterologist; these interventions were not randomized or controlled.

\section{EN Group}

The EN group has been described elsewhere [22]; in brief, gastroenterologists referred patients for EN therapy who met the following criteria: 16-40 years of age, newly diagnosed with CD involving the small intestine, interested in using EN therapy to treat active disease, and no corticosteroid therapy in the last 2 weeks. Patients received either 8 weeks of EEN or 2 weeks of EEN followed by 6 weeks of EN plus 1 small meal of usual food per day. Both treatments aimed to induce disease remission, and both required patients to forgo usual food and fluids except water and black tea or coffee for at least 2 weeks if prescribed partial EN or 8 weeks if prescribed EEN. Standard care maintenance therapies after the period of $\mathrm{EN}$ were prescribed at the discretion of the treating gastroenterologist.

\section{Quality and Disease Activity Assessments}

Patient demographics and information on current medication use were collected at baseline and 6 months. HRQOL was assessed using the validated short IBDQ (SIBDQ) [23]. The SIBDQ is a short form of the 32-question IBDQ and includes questions from each of the 4 domains of the IBDQ. The SIBDQ explains $92 \%$ of the variance in the full IBDQ, and clinical changes in disease activity are reflected in SIBDQ score variations [23]. The SIDBQ asks 14 questions related to the impact of disease symptoms on social and personal functioning. The answer to each question is assigned a score of 1-7 points, the sum of which is the SIBDQ score. For each question, a score of 1 indicates very poor HRQOL, and a score of 7 indicates optimal HRQOL. A clinically significant HRQOL response is a SIBDQ of $>47$ points [23].

The presence of anxiety and depression were assessed using the HADS questionnaire, which is composed of 14 specific questions [24]. A HADS subscale score of $<8$ suggests the absence of depression or anxiety; a score of 8 to 10 suggests borderline anxiety or depression, and a score of $\geq 11$ suggests the presence of moderate to severe anxiety or depression [24]. The sensitivity and specificity of the HADS to identify cases of anxiety and depression is approximately 0.80 [25].

Disease activity was assessed with the Harvey-Bradshaw Index (HBI) [26]. The assessment of extraintestinal manifestations and abdominal mass were completed using the patient hospital notes.

\section{Standard Care Group}

The standard care group completed the questionnaires immediately after diagnosis (baseline) and after 6 months. They were sent the questionnaires by post and were instructed to return them 
Table 1. Baseline characteristics of patients with newly diagnosed Crohn's disease who were offered enteral nutrition (EN) or standard care (SC) to induce disease remission

\begin{tabular}{|c|c|c|c|c|}
\hline & $\begin{array}{l}\text { SC group } \\
(n=22)\end{array}$ & $\begin{array}{l}\text { Completed EN } \\
\text { treatment } \\
(n=18)\end{array}$ & $\begin{array}{l}\text { Did not complete } \\
\text { EN treatment } \\
(n=12)\end{array}$ & $\begin{array}{l}p \text { value } \\
\text { SC vs. } \\
\text { completed EN }\end{array}$ \\
\hline Age, years & $30.5[19-71]$ & $23[16-37]$ & 16.5 [15-39] & 0.007 \\
\hline $\begin{array}{l}\text { Harvey-Bradshaw Index } \\
\text { Medication }\end{array}$ & $5[0-19]$ & $4.5[0-12]$ & $6[0-14]$ & 0.741 \\
\hline None & $1(5)$ & $9(50)$ & $5(45)$ & \\
\hline Mesalazine & $20(91)$ & $6(33)$ & $5(45)$ & \\
\hline Azathioprine/6-mercaptopurine & $11(50)$ & $2(11)$ & $1(9)$ & \\
\hline $\begin{array}{l}\text { SIBDQ } \\
\text { Hospital Anxiety and Depression Score }\end{array}$ & $45[19-69]$ & $37.5[15-63]$ & $42.0[15-54]$ & 0.026 \\
\hline HADS anxiety score & $4.0[0-12]$ & $7.5[0-15])$ & $6.0[1-14]$ & 0.084 \\
\hline Anxiety (HADS-A >10) & $1(9)$ & $5(28)$ & $3(25)$ & \\
\hline Borderline anxiety (HADS-A 8-10) & $5(22)$ & $4(22)$ & $2(18)$ & \\
\hline HADS depression score & $2.0[0-13]$ & $4.0[0-10]$ & $4.5[2-14]$ & 0.484 \\
\hline Depression (HADS-D >10) & $1(5)$ & $0(0)$ & $2(18)$ & \\
\hline Borderline depression (HADS-D 8-10) & $3(14)$ & $2(11)$ & $2(18)$ & \\
\hline
\end{tabular}

Values are medians [ranges] or numbers (\%). HADS, Hospital Anxiety and Depression Score; SIBDQ, Short Inflammatory Bowel Disease Questionnaire.

within 1 week, but questionnaires were accepted if returned within 1 month. Patients may have commenced medical treatment at the time of completing the baseline questionnaires.

\section{EN Group}

The EN group completed the questionnaires during a research appointment at the hospital. Questionnaires were completed at baseline prior to starting treatment with $\mathrm{EN}$, fortnightly during the 8-week EN treatment, and at 6 months.

\section{Statistics}

The results are presented as percentages, medians, and ranges. Nonparametric statistical tests were used due to nonnormal distribution of many variables. Differences between treatment groups were compared using the Mann-Whitney U test or Fisher's exact test, and within group changes were assessed using the Friedman test or Wilcoxon matched-pair signed-rank test. Correlations between variables were assessed using Spearman's rank correlation. Statistical significance was present with $p<0.05$. Statistical tests and graphs were prepared in Prism 7 version 7.03 (GraphPad Software, Inc., La Jolla, CA, USA).

\section{Results}

\section{Baseline Characteristics of the Study Groups}

Twenty-seven patients with newly diagnosed CD were recruited to the standard care group; complete data at both time points were available for $22(81 \%)$ patients. Thirty patients with newly diagnosed CD were recruited to use $\mathrm{EEN}(n=21)$ or partial EN $(n=8)$ to induce disease remission. EN treatment and follow-up to 6 months were completed by $18(60 \%)$ patients. Twelve (40\%) patients did not complete EN treatment, or did not respond ( $n=$ 2) to EN treatment, and were consequently withdrawn from the research study. At baseline, patients who did and did not complete EN treatment had similar psychological characteristics. However, patients who tolerated EN and completed the treatment were more likely to have finished secondary school education than patients who did not complete EN treatment ( 11 vs. $67 \%$, respectively; $p=$ 0.004).

Overall, the patients who used EN treatment to induce disease remission were younger, had lower HRQOL, and higher levels of anxiety, but similar depression and disease activity scores compared with those who used standard care to treat active newly diagnosed CD (Table 1). Patients recruited to use standard care were more likely to have started on treatment when they completed the baseline questionnaires (Table 1). At baseline, the SIBDQ and disease activity score of all patients $(n=40)$ were not significantly correlated $(p>0.05)$.

\section{Psychological Outcomes during the First 6 Months after Diagnosis}

HRQOL improved significantly in all patients, regardless of treatment, during the first 6 months (Fig. 1). In the EN group, HRQOL improved significantly $(p<0.0001)$ after 2 weeks of exclusive EN. Six months after diagnosis, 


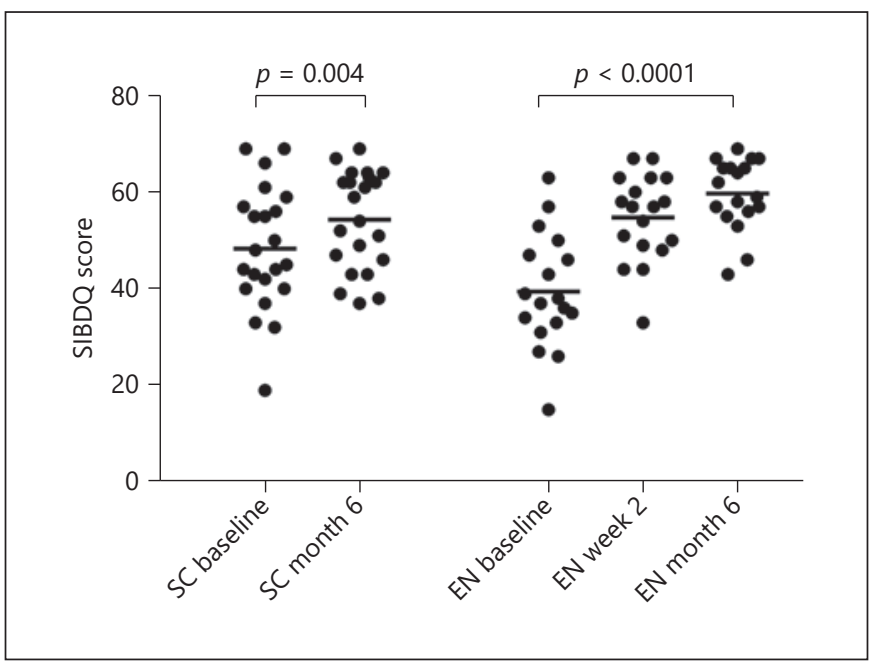

Fig. 1. Short inflammatory bowel disease questionnaire (SIBDQ) scores of patients at baseline, and 2 weeks and 6 months after diagnosis of Crohn's disease treated with standard care (SC; $n=22)$ or enteral nutrition ( $\mathrm{EN} ; n=18)$.

there was no significant difference in the median SIBDQ scores between those who had used standard care or EN to induce disease remission. A clinically significant SIBDQ score of $>47$ was present in a greater proportion of patients at 6 months $(31 / 40[78 \%])$ than at baseline $(15 / 40[38 \%] ; p=0.006)$. At month 6 , but not at baseline, SIBDQ score was moderately correlated $(\mathrm{r}=-0.58,95 \%$ CI -0.76 to $-0.31 ; p<0.0001)$ with $\mathrm{HBI}$ disease activity score.

HADS anxiety scores improved significantly within 2 weeks of EN treatment $(p=0.002)$ and continued to improve during the first 6 months after diagnosis (Fig. 2). Anxiety scores did not significantly change in patients who used standard care $(p>0.05)$. Anxiety scores at 6 months were not significantly different between those who used standard care or EN to induce disease remission $(p>0.05)$.

HADS depression scores in the EN group improved during the first 6 months of treatment (Fig. 3). There was no significant change in HADS depression scores from baseline to month 6 in patients on standard care treatment $(p>0.05)$. Scores at 6 months were not significantly different between those who used standard care or EN $(p>0.05)$.

\section{Psychological Outcomes of Patients Who Did Not \\ Complete EN Treatment}

Psychological outcome data for the 12 patients who did not complete EN treatment were collected up until

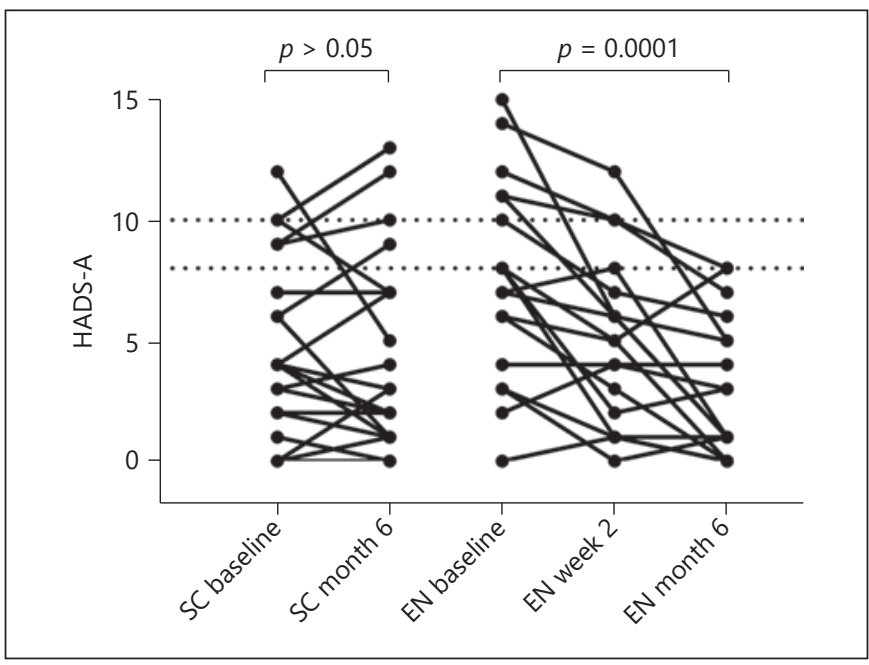

Fig. 2. Anxiety subscores of the Hospital Anxiety and Depression Score (HADS-A) at baseline, and 2 weeks and 6 months after diagnosis of Crohn's disease in patients treated with standard care (SC; $n=22)$ or enteral nutrition (EN; $n=18)$.

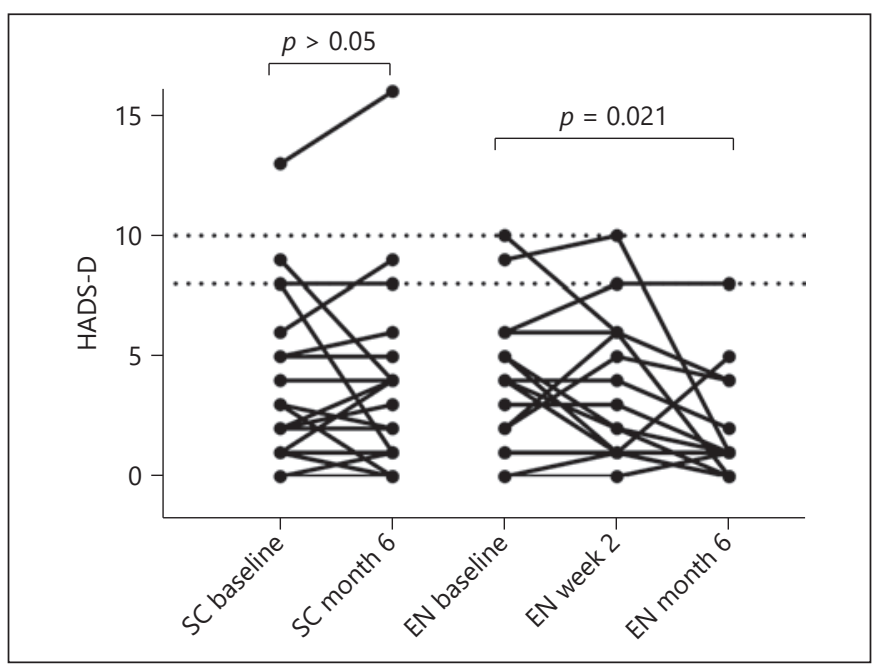

Fig. 3. Depression subscores of the Hospital Anxiety and Depression Score (HADS-D) at baseline, and 2 weeks and 6 months after diagnosis of Crohn's disease in patients treated with standard care (SC; $n=22$ ) or enteral nutrition (EN; $n=18)$.

patients withdrew from the study. Four patients did not manage to initiate EEN, either because they decided to use steroids instead $(n=2)$ or due to intolerance of the enteral formula $(n=2)$, and withdrew within the first week of the study. Follow-up data were available for 8 patients at week 2 and 6 patients at week 4 . Eight patients
10

Inflamm Intest Dis 2019;4:7-13 DOI: $10.1159 / 000497323$
Wall/McCombie/Gearry/Day 
successfully initiated EEN: at week 2, HRQOL had improved in 4 of the 8 patients, remained the same in 2 patients, and declined in the 2 patients who were withdrawn after week 2 due to nonadherence to the protocol. Improvements in HADS scores were also observed in all but 1 patient. At week 4, initial improvements in psychological outcomes were not sustained, and 5 of the 6 patients had deterioration in HRQOL, anxiety scores, or depression scores. These 6 patients were withdrawn from the study due to lack of response $(n=2)$, newly developed intolerance to $\mathrm{EN}(n=2)$, bowel perforation $(n=1)$, and patient decision to not reinsert a nasogastric EN feeding tube $(n=1)$. At week 4 , psychological outcomes of this patient group were similar to those at baseline.

\section{Treatments 6 Months after Diagnosis}

At 6 months, patients in the standard care group were on 5-aminosalicyclic acid ( $n=14$ [64\%]), corticosteroids $(n=1[5 \%])$, azathioprine $(n=8[36 \%])$, or adalimumab $(n=2[9 \%])$. The median HBI had improved from 5 at baseline to 3 (range, $0-21)$ at 6 months $(p=0.049)$, and $17 / 22$ $(77 \%)$ of patients were in clinical remission $(\mathrm{HBI}<5)$.

Patients who used EN to induce disease remission were on 5-aminosalicyclic acid ( $n=3$ [17\%]), corticosteroids $(\mathrm{n}=1[6 \%])$, azathioprine or 6 -mercaptopurine $(n=11[61 \%])$, or adalimumab $(n=3[17 \%])$. The median HBI had improved from 4.5 prior to treatment initiation to 2 (range, $0-8$ ) at 6 months $(p=0.005$ ), and $17 / 18$ $(94 \%)$ of patients were in clinical remission $(\mathrm{HBI}<5)$.

\section{Discussion}

The prevalence of anxiety and depression and the HRQOL of patients with newly diagnosed CD is not well described in the literature, neither is how these outcomes may change during the months following diagnosis. It is known that poor HRQOL is associated with active disease $[3,5,23,27-29]$, which was also apparent in this study. HRQOL improved significantly once treatment was initiated, suggesting that improvements in HRQOL were related to reduced symptoms of active disease.

There is evidence that some IBD treatments may impact on HRQOL more than others [4-7, 12, 13]. Exclusive $\mathrm{EN}$ to induce disease remission has been associated with improved HRQOL in children $[19,29]$ and Chinese adults with CD [30], but its impact on HRQOL has not been reported in adults in western countries. A pediatric study found that children who used steroids have a slightly higher QOL score than children who used EEN to induce

Newly Diagnosed Crohn's Disease:

Psychological Outcomes over 6 Months remission [29]. However, they did not report QOL scores once children had resumed eating a usual diet. Our data show that at 6 months, $78 \%$ of patients who used standard care or EN to induce disease remission had a clinically significant SIBDQ score, and there was no difference in HRQOL scores of patients, which may be expected given that the majority of patients were in remission at that point in time.

Anxiety is reported to be present in up to $40 \%$ of patients with IBD $[11,14,31]$ and is more likely to be present in patients with active disease [13, 14, 16, 17]. There are few reports of how anxiety may change over time or with change in disease activity. Anxiety was present in $48 \%$ of patients with newly diagnosed CD referred for EN treatment and in $31 \%$ of newly diagnosed patients in the standard care group. At baseline, there was a trend towards higher HADS anxiety scores in patients referred for EN, which may be attributed to higher disease activity because they were awaiting treatment rather than anxiety related to EN treatment. Once EN treatment was initiated, anxiety scores improved significantly and were more comparable with the baseline scores in the standard care group, of which many had already started treatment when assessed. At 6 months, anxiety was still present in a proportion of patients in concordance with the existing literature $[10,14]$.

The presence of depression in IBD cohorts varies in the literature from 4 to $22 \%$ of patients $[2,9,13,14]$. Previous research has found that the presence of depression is more likely in patients with symptoms of active disease $[13,14,16]$. Prior to starting EN treatment, depression was present in $15 \%$ of patients with newly diagnosed CD. The presence of depression decreased during the first 6 months in both treatment groups, but a proportion of patients still had symptoms of depression at 6 months. The limited literature [10] which describes depressive symptoms over time suggests that the persistence of depression despite treatment of active disease is not uncommon.

\section{Limitations and Future Directions}

This research has some limitations. The two patient cohorts were recruited in different time periods, and, therefore, their treatments may reflect the differences in service delivery or drug availability at that time. The initial improvements in psychological outcomes on EN cannot be compared directly with the standard care group because the baseline assessments were not completed prior to treatment initiation in all patients who received standard care to induce disease remission. $\mathrm{Pa}$ tients who received EN therapy received multiple dieti- 
tian follow-up appointments during the study, whereas the standard care group did not. This variation in patient follow-up and support may have had some effect on psychological outcomes at 6 months. In the standard care group, $81 \%$ of patients had baseline and follow-up data, whereas complete data were available for only $18 / 30$ (60\%) patients referred for EN therapy. The inclusion of only patients who completed EN treatment may not be a true representation of the psychological outcomes of patients interested in using EN to induce disease remission.

A proportion of patients in the $\mathrm{EN}$ group did receive standard care maintenance treatments during EN treatment or after EN completion. These treatments were prescribed at the discretion of the gastroenterologist based on best practice guidelines at the time. Retrospective pediatric cohort studies show that early introduction of thiopurine medications during EEN treatment is common practice $[32,33]$. Reports of EEN as an adjuvant therapy in adult patients with mild-to-moderate CD are lacking. Therefore, the EN group was not strictly on EN for 6 months, which needs to be taken into account in interpreting the results.

It is possible that patients who chose EN therapy may have a high expected value for this therapy and so may be susceptible to a placebo effect. The data of patients who did not complete EN therapy show that initial improvements in psychological outcomes occur within 2 weeks of starting EN and suggest that HADS anxiety and HRQOL scores may be sensitive to changes in either patient perception or actual efficacy of EN treatment. Future studies could measure pretreatment expectations of patients starting EN therapy and usual care treatments and link this to responsiveness, especially in the psychological domains. Most importantly, future research should use a direct comparison group.

\section{Conclusion}

This research suggests that the presence of anxiety and depression in New Zealand adults with active CD is equivalent to those previously reported internationally. In this unique cohort of newly diagnosed CD patients with active disease, induction of remission treatment with EN resulted in significant reductions in anxiety and depression scores, and clinically significant improvements in the HRQOL; at 6 months, these outcomes were similar to those of patients treated with standard care. The use of EN therapy, instead of standard care, to induce disease remission results in similar longitudinal improvements in psychological outcomes as standard care treatments. Future research should address this with a direct comparison group.

\section{Acknowledgments}

We would like to acknowledge the intellectual contribution of Prof. Roger T. Mulder to the standard care study.

\section{Statement of Ethics}

Subjects gave their written informed consent. The study protocol was approved by the research institute's committee on human research.

\section{Disclosure Statement}

The authors have no conflicts of interest to disclose.

\section{Funding Sources}

Research with the EN cohort was supported by funding from the New Zealand Society of Gastroenterology, Dietitians NZ, Australasian Society of Parenteral and Enteral Nutrition, NZ Federation of Graduate Women, the Maurice and Phyllis Paykel Trust and a Fanny Evans Postgraduate Scholarship for Women. A.M. McCombie is the recipient of the University of Otago Doctoral Scholarship and Todd Foundation award for excellence.

\section{Author Contributions}

C.L.W. collected and analyzed the EN cohort data and drafted the manuscript. A.M.M. collected and analyzed the standard care cohort data, and reviewed and approved the final manuscript. R.B.G. provided intellectual support for the EN and standard care cohort studies, and reviewed and approved the final manuscript. A.S.D. provided intellectual support for the EN cohort study, and reviewed and approved the final manuscript.

\section{References}

1 Blondel-Kucharski F, Chircop C, Marquis P, Cortot A, Baron F, Gendre JP, et al.; Groupe d'Etudes Thérapeutique des Affections Inflammatoires Digestives (GETAID). Healthrelated quality of life in Crohn's disease: a prospective longitudinal study in 231 patients. Am J Gastroenterol. 2001 Oct;96(10):2915-20.

2 Iglesias-Rey M, Barreiro-de Acosta M, Caamaño-Isorna F, Rodríguez IV, Ferreiro R, Lindkvist B, et al. Psychological factors are associated with changes in the health-related quality of life in inflammatory bowel disease. Inflamm Bowel Dis. 2014 Jan;20(1):92-102.
12

Inflamm Intest Dis 2019;4:7-13

DOI: $10.1159 / 000497323$
Wall/McCombie/Gearry/Day 
3 van der Have M, van der Aalst KS, Kaptein AA, Leenders M, Siersema PD, Oldenburg B, et al. Determinants of health-related quality of life in Crohn's disease: a systematic review and meta-analysis. J Crohn's Colitis. 2014 Feb;8(2):93-106.

4 Loftus EV, Feagan BG, Colombel JF, Rubin DT, Wu EQ, Yu AP, et al. Effects of adalimumab maintenance therapy on health-related quality of life of patients with Crohn's disease: patient-reported outcomes of the CHARM trial. Am J Gastroenterol. 2008 Dec;103(12): 3132-41. Erratum in: Am J Gastroenterol. 2009 Jul;104(7):1894.

5 Feagan BG, Yan S, Bala M, Bao W, Lichtenstein GR. The effects of infliximab maintenance therapy on health-related quality of life. Am J Gastroenterol. 2003 Oct;98(10):2232-8.

6 Bernklev T, Jahnsen J, Schulz T, Sauar J, Lygren I, Henriksen M, et al. Course of disease, drug treatment and health-related quality of life in patients with inflammatory bowel disease 5 years after initial diagnosis. Eur J Gastroenterol Hepatol. 2005 Oct;17(10): 1037-45.

7 Haapamäki J, Turunen U, Roine RP, Färkkilä MA, Arkkila PE. Impact of demographic factors, medication and symptoms on diseasespecific quality of life in inflammatory bowel disease. Qual Life Res. 2009 Oct;18(8):961-9.

8 Kalafateli M, Triantos C, Theocharis G, Giannakopoulou D, Koutroumpakis E, Chronis A, et al. Health-related quality of life in patients with inflammatory bowel disease: a singlecenter experience. Ann Gastroenterol. 2013; 26(3):243-8.

9 Goodhand JR, Wahed M, Mawdsley JE, Farmer AD, Aziz Q, Rampton DS. Mood disorders in inflammatory bowel disease: relation to diagnosis, disease activity, perceived stress, and other factors. Inflamm Bowel Dis. 2012 Dec;18(12):2301-9.

10 Graff LA, Walker JR, Bernstein CN. Depression and anxiety in inflammatory bowel disease: a review of comorbidity and management. Inflamm Bowel Dis. 2009 Jul;15(7): 1105-18.

11 Mikocka-Walus AA, Turnbull DA, Andrews JM, Moulding NT, Holtmann GJ. The effect of functional gastrointestinal disorders on psychological comorbidity and quality of life in patients with inflammatory bowel disease. Aliment Pharmacol Ther. 2008 Aug;28(4): 475-83.

12 Baig BJ, Lees CW, Johnstone EC, James V, Satsangi J, Lawrie SM. W09-03. Associations of affective disorder in patients with inflammatory bowel disease. Eur Psychiatry. 2011; 26 Suppl 1:2220.
13 Nahon S, Lahmek P, Durance C, Olympie A, Lesgourgues B, Colombel JF, et al. Risk factors of anxiety and depression in inflammatory bowel disease. Inflamm Bowel Dis. $2012 \mathrm{Nov}$; 18(11):2086-91.

14 Tribbick D, Salzberg M, Ftanou M, Connell WR, Macrae F, Kamm MA, et al. Prevalence of mental health disorders in inflammatory bowel disease: an Australian outpatient cohort. Clin Exp Gastroenterol. 2015 Jul;8:197204.

15 Chan W, Shim HH, Lim MS, Sawadjaan FL, Isaac SP, Chuah SW, et al. Symptoms of anxiety and depression are independently associated with inflammatory bowel disease-related disability. Dig Liver Dis. 2017 Dec;49(12): 1314-9.

16 Banovic I, Gilibert D, Cosnes J. Crohn's disease and fatigue: constancy and co-variations of activity of the disease, depression, anxiety and subjective quality of life. Psychol Health Med. 2010 Aug;15(4):394-405.

17 Byrne G, Rosenfeld G, Leung Y, Qian H, Raudzus J, Nunez C, et al. Prevalence of Anxiety and Depression in Patients with Inflammatory Bowel Disease. Can J Gastroenterol Hepatol. 2017;2017:6496727.

18 Persoons P, Vermeire S, Demyttenaere K, Fischler B, Vandenberghe J, Van Oudenhove $\mathrm{L}$, et al. The impact of major depressive disorder on the short- and long-term outcome of Crohn's disease treatment with infliximab. Aliment Pharmacol Ther. 2005 Jul;22(2): 101-10.

19 Afzal NA, Van Der Zaag-Loonen HJ, ArnaudBattandier F, Davies S, Murch S, Derkx B, et al. Improvement in quality of life of children with acute Crohn's disease does not parallel mucosal healing after treatment with exclusive enteral nutrition. Aliment Pharmacol Ther. 2004 Jul;20(2):167-72.

20 Takagi S, Utsunomiya K, Kuriyama S, Yokoyama H, Takahashi S, Iwabuchi M, et al. Effectiveness of an 'half elemental diet' as maintenance therapy for Crohn's disease: A randomized-controlled trial. Aliment Pharmacol Ther. 2006 Nov;24(9):1333-40.

21 McCombie AM, Mulder RT, Gearry RB. Coping Strategies and Psychological Outcomes of Patients with Inflammatory Bowel Disease in the First 6 Months After Diagnosis. Inflamm Bowel Dis. 2015 Oct;21(10):2272-80.

22 Wall CL, Gearry RB, Day AS. Treatment of Active Crohn's Disease with Exclusive and Partial Enteral Nutrition: A Pilot Study in Adults. Inflamm Intest Dis. 2018 Jul;2(4): 219-27.
23 Irvine EJ, Zhou Q, Thompson AK. The Short Inflammatory Bowel Disease Questionnaire: a quality of life instrument for community physicians managing inflammatory bowel disease. CCRPT Investigators. Canadian Crohn's Relapse Prevention Trial. Am J Gastroenterol. 1996 Aug;91(8):1571-8.

24 Zigmond AS, Snaith RP. The hospital anxiety and depression scale. Acta Psychiatr Scand. 1983 Jun;67(6):361-70.

25 Bjelland I, Dahl AA, Haug TT, Neckelmann D. The validity of the Hospital Anxiety and Depression Scale. An updated literature review. J Psychosom Res. 2002 Feb;52(2):69-77.

26 Harvey RF, Bradshaw JM. A simple index of Crohn's-disease activity. Lancet. 1980 Mar; 1(8167):514.

27 Gibson PR, Weston AR, Shann A, Florin TH, Lawrance IC, Macrae FA, et al. Relationship between disease severity, quality of life and health-care resource use in a cross-section of Australian patients with Crohn's disease. J Gastroenterol Hepatol. 2007 Aug;22(8): 1306-12.

28 Wright EK, Kamm MA, De Cruz P, Hamilton AL, Ritchie KJ, Krejany EO, et al. Effect of intestinal resection on quality of life in Crohn's disease. J Crohn's Colitis. 2015 Jun;9(6):45262.

29 Hart L, Farbod Y, Halgren CR, Mbuagbaw L, Zachos M, Pai N. Measuring quality of life and disease activity in pediatric patients receiving induction therapy of exclusive enteral nutrition or corticosteroids for active inflammatory bowel disease. J Canadian Assoc Gastroenterol. 2018;1 Suppl 1:264-5.

30 Guo Z, Wu R, Zhu W, Gong J, Zhang W, Li Y, et al. Effect of exclusive enteral nutrition on health-related quality of life for adults with active Crohn's disease. Nutr Clin Pract. 2013 Aug;28(4):499-505.

31 Selinger CP, Lal S, Eaden J, Jones DB, Katelaris P, Chapman G, et al. Better disease specific patient knowledge is associated with greater anxiety in inflammatory bowel disease. J Crohn's Colitis. 2013 Jul;7(6):e214-8.

32 Grover Z, Lewindon P. Two-Year Outcomes After Exclusive Enteral Nutrition Induction Are Superior to Corticosteroids in Pediatric Crohn's Disease Treated Early with Thiopurines. Dig Dis Sci 2015 Oct;60(10):3069-74.

33 Frivolt K, Schwerd T, Werkstetter KJ, Schwarzer A, Schatz SB, Bufler P, et al. Repeated exclusive enteral nutrition in the treatment of paediatric Crohn's disease: predictors of efficacy and outcome. Aliment Pharmacol Ther. 2014 Jun;39(12):1398-407.
Newly Diagnosed Crohn's Disease:

Psychological Outcomes over 6 Months
Inflamm Intest Dis 2019;4:7-13

DOI: $10.1159 / 000497323$ 